\title{
Conditions for noise reduction and stable encoding of spatial structure by cortical neural networks.
}

\author{
Boris S. Gutkin ${ }^{1}$ and Charles E. Smith²
}

${ }^{1}$ Center for Neural Basis of Cognition and Department of Mathematics, University of Pittsburgh, Pittsburgh, Pa 15217, email: gutkin@ @nbc.cmu.edu

2Biomathematics Programme, Department of Statistics, North Carolina State University, Raleigh, NC 27695, email: cesmith@stat.ncsu.edu

\section{Introduction.}

Several works have suggested that cortical sensory networks are robust encoders that act to reduce the uncertainty in the stimuli by reducing noise (Douglas et al. 1995) while accentuating the spatial structure of the stimulus (Shamma 1989). In a recent report Amit and Brunel (1997) suggest that stimulus encoding ability of the cortex (e.g. the prefrontal cotrex coding with attractors) takes place in the conext of high spontaneous cortical activity. The authors further suggest that given a relative balance between excitatory and inhibitory inputs impinging on a single neuron, the stochastic background is stable. In this work we seek to address the issue of robust encoding of spatialy structured stimuli by recurrent "cortical" neural networks. To do so we develop mathematical conditions for stability of such neural networks when the spatially structured stimulus is corrupted by additive noise. 
In particular we consider neural networks of the Wilson-Cowan type with local connections designed to reflect the lateral connectivity seen in the primary sensory cortices (e.g. SI - somatosensory cortex). The input to the network consists of a temporally stationary spatial pattern of excitation perturbed by additive noise. Our goal is to present conditions on the parameters of the network that guarantee that the spatial structure in the stimulus is robustly encoded by the network. More specifically we seek a network that encodes the salient features of a noisy input pattern and reaches a statistically stable stationary distribution of activity. We shall show that our analytical conditions are consistent with the near balance hypothesis.

Previous work has shown that deterministic neural networks with lateral inhibitory connection patterns under some conditions act as spatial band-pass filters capable of contrast enhancement (Shamma 1989). Such a network can act to reduce the amount of data necessary to encode structure in the stimulus. Furthermore a large body of literature considered the stability of deterministic recurrent neural networks particularly in the context of attractor neural networks (e.g. Cohen and Grossberg 1983, Kishimoto and Amari 1979, Ermentrout 1982, Markus and Westervelt 1989). Cohen and Grossberg (1983) developed a general condition for global asymptotic stability of attractor state in a deterministic recurrent neural network using the Lyapunov function approach. As will be seen below we use a modified Lyapunov function method to show the stability of moments of activity for a cortical neural network under a noisy stimulus. Thus our result extends previous mean stability results to higher order moments: the temporal variance and spatial covariance of the network activity in particular. The conditions we develop are closely related to the conditions developed by Markus and 
Westerveldt (1989) for a recurrent neural network with delay. To summarize, our goal is to give quantitative conditions on the connection matrix and the sigmoid transfer function that would guarantee that the network responds to the stimulus by a spatially structured steady state with a variance which is less than that of the input.

Previous efforts that have considered the influence of additive noise on cortical neural networks either did not have spatial structure (e.g. Ohura and Cowan 1995) or did not provide much in the way of formal analysis (e.g. Buhmann 1987), whereas the large literature that looked at stability of stochastic artificial neural networks treated primarily the case where the noise was of synaptic origin, i.e. random fluctuations in the weights or parametric multiplicative noise. Here we study a spatially structured network under noisy data conditions and analyze the dynamics of the covariance as well as the mean. We first provide some general conditions guaranteeing stochastic stability of the steady state response. Then we apply linear stability analysis to arrive at a condition on the connection matrix that ensures local stability of the mean pattern and a bounded stationary covariance structure. We further show that for a appropriate choice of the connection weights, the network acts to reduce the variance on the input pattern.

\section{Model Description.}

The initial motivation for this work is a biological one. The network in question has been proposed as a model for information dynamics in the primary somatosensory cortex. It is based on the neurophysiological studies (Juliano et al. 1981) that showed that the cortical response to a constant tactile stimulus in the primary somatosensory cortex (SI) had considerable spatial inhomogeneity not predicted by anatomical structure. Their 
hypothesis was that the cortical dynamics act on the stimulus to accentuate certain spatial frequencies while reducing the amplitude of others. In fact the cortical dynamics filter out the spatialy constant inputs and enhance the spatial contrasts in the stimulus. Specifically for the experimental results in (Juliano et al. 1981), Whitsel et al. (1988) proposed that the cortical lateral inhibitory network in the SI tends to accentuate the spatial discontinuities in the sensory input from the thalamus, unmasking the spatial structure of the tactile stimulus while reducing the number of units active. That is the cortical network reaches a stationary state of activity where only the units corresponding to the location of the edges and corners are active. Extensive numerical work on the deterministic version of a deterministic network model designed to model the cortical dynamics that underlie formation of spatial patterns was carried out in (Smith and Kelly 1985 and Whitsel and Kelly 1988) and formal stability analysis was presented in (Kelly 1990). A preliminary version of this work was presented in (Gutkin and Smith 1994).

The model we consider is a discrete-space, continuous-time version of networks first described by Cowan (1970), for which extensive analytical literature exists (e.g. Cowan 1970, Ermentrout 1982). In our case the network is a 2-dimensional grid or a 1dimensional array of sparsely connected sigmoidal neural units whose state is characterized an activation variable $\mathrm{x}$. This variable is allowed to take both positive and negative values, reflecting excitatory and inhibitory activity within a neural aggregate, e.g. a cortical mini-column. The network equations are the following system of nonlinear Langevin equations:

$$
\mu \dot{x}_{j}(t)=-x_{j}(t)+\sum_{k=1}^{N} w_{j k} f_{k}\left(x_{k}(t)\right)+p_{j}+\sigma \dot{\xi}_{j} j=1 \ldots N
$$


Here $f_{k}\left(x_{k}\right): R->(-1,1)$ is a sigmoid non-linearity giving the normalized firing rate of the neural aggregate; $w_{j k}$ is the connection weight from unit $k$ to unit $j ; p_{j}$ 's represents the time invariant spatially structured inputs and $x_{j}$ is the noise term, and $\sigma$ is the scale parameter of the noise. From now on we set $\tau=1$ without loss of generality. For notational convenience we may write system (2.1) in vector matrix form:

(2.2) $\quad \dot{\vec{x}}=-\vec{x}(t)+\vec{p}+W F(\vec{x}(t))+\sigma \vec{N}(t)$

with

$$
\begin{aligned}
& \overrightarrow{\mathrm{x}}=\left[\begin{array}{lllll}
\mathrm{x}_{1}(\mathrm{t}) & \mathrm{x}_{2}(\mathrm{t}) & \ldots & \mathrm{x}_{\mathrm{N}}(\mathrm{t})
\end{array}\right]^{\mathrm{T}} \quad \overrightarrow{\mathrm{p}}=\left[\begin{array}{lllll}
\mathrm{p}_{1} & \mathrm{p}_{2} & \ldots & \mathrm{p}_{\mathrm{N}}
\end{array}\right]^{\mathrm{T}} \\
& \mathrm{N}(\mathrm{t})=\left[\begin{array}{llllll}
\dot{\xi}_{1}(\mathrm{t}) & \dot{\xi}_{2}(\mathrm{t}) & \ldots & \dot{\xi}_{\mathrm{N}}(\mathrm{t})
\end{array}\right]^{\mathrm{T}} \quad \mathrm{F}(\overrightarrow{\mathrm{x}}(\mathrm{t}))=\left[\begin{array}{llll}
\mathrm{f}_{1}\left(\mathrm{x}_{1}\right) & \mathrm{f}_{2}\left(\mathrm{x}_{2}\right) & \ldots & \mathrm{f}_{\mathrm{k}}\left(\mathrm{x}_{\mathrm{k}}\right)
\end{array}\right]^{\mathrm{T}} \\
& \mathrm{W}=\left(\begin{array}{ccc}
\mathrm{w}_{11} & \ldots & \mathrm{w}_{1 N} \\
\vdots & \ddots & \vdots \\
\mathrm{w}_{\mathrm{N} 1} & \ldots & \mathrm{w}_{N N}
\end{array}\right)
\end{aligned}
$$

Numerical simulations serve to illustrate the behvavior of a 2-dimensional version of system (2.1). A constant spatially structured stimulus was delivered at every iteration to a 2-dimensional network (2.1), see fig 1a. The stimulus was corrupted by a noise simulated at each location by sampling independent Gaussian random deviates with a preset variance. We studied a homogeneous network with "Mexican hat" connection patterns shown in fig $1 \mathrm{~b}$ and $1 \mathrm{c}$. The main feature seen in the simulations of the network weak lateral inhibition is the formation of an apparent steady state response despite the presence of noise, fig $2 \mathrm{a}$. The network clearly encoded edges with random fluctuations weakly perturbing the activity of single units. Further simulations suggested 
that the steady state response is independent of the initial conditions of the network. The mean steady state pattern could be easily recovered by temporal averaging.

On the other hand a network with strong lateral inhibition (fig 1c) did not reach a steady state, or at least, there was no steady state with any discernible spatial features of the stimulus, fig $2 \mathrm{~b}$. The pattern of activity lost all resemblance to the stimulus under noisy conditions dramatically faster than the corresponding deterministic system. Furthermore, temporal or spatial averaging did not recover any stimulus dependent spatial structure and there is no apparent trend in the dynamics of a single unit, save for an increasing variance. Thus numerical results suggested that strong lateral inhibition leads to a network that is highly sensitive to noise and does not act as a stable encoder of spatial structure. Thus we set out to provide analytic conditions on the lateral connections matrix that yield steady state response that not strongly influenced by the additive noise.

3. Stochastic Stability of the Sigmoid Network.

We now construct conditions under which the network (2.2) has a unique attractive stationary distribution parametrized by the input. Here we apply the method of stochastic Lyapunov functions to the network (2.2) written as a system of non-linear Ito stochastic differential equations:

$$
d x=(-x(t)+p+W x(t)) d t+\sigma I d W_{t}
$$

where $\mathrm{x}$ is a N-vector, $\mathrm{dW}_{\mathrm{t}}$ is an M-vector of $\delta$-correlated Gaussian deviates and $\sigma^{2}$ is variance of the noise. We first state the theorem that underlies our result: 
Theorem 1.(Gard 1988)

Consider the stochastic differential equation (s.d.e) of the form:

$$
d X=f(X, t) d t+G(x, t) d W_{t}
$$

where $X$ is a N-vector, $f() \quad N$-vector of functions describing the deterministic drift, $W_{t}$ is an $M$-vector of delta-correlated Gaussian noises and $G()$ is a NxMmatrix of variances

Let there be a continuous function $\mathrm{V}(\mathrm{x}): \mathrm{R}^{\mathrm{n}}-\mathrm{R}$ s. $\mathrm{t}$.

(i) $V(x)$ is non-negative and $C^{2}$ for all $x$ not 0 with $V(x)=0$ for $\|x\|=0$

Define the differential operaton $L V \equiv \frac{d V}{d t}+\nabla V \cdot f+\frac{1}{2} \operatorname{tr}\left(G V_{x x} G^{T}\right)$

(ii) If $L V<0$ for $|x|>0$, then the solution of (3.2) is statistically stable.

The function $V(t, x)$ in the theorem is known as a stochastic Lyapunov function. Next we exhibit such a function for (3.1) together with appropriate conditions.

Theorem 2.

Let $V(x)=|x|$ where the $\mid . . . I$ denotes the usual $L_{1}$-norm

Define IIWII as the real number $\max _{|x|} \frac{|\mathrm{Wx}|}{|\mathrm{x}|}$

If (i) $\left[F^{\prime}\left(x_{i}\right)\right] i<B$ for all $x$ where $F^{\prime}$ is the Jacobian of $F$ evaluated at $x$ and BIIWII $<1$, 
Then criteria for the Theorem are satisfied and $V(x)$ is a stochastic Lyapunov function.

Proof:

A detailed proof is published in Gutkin (1993), here we present a brief sketch of it. Without loss of generality we consider the stability of the homogenous solution. Once the homogeneous solution is proven to be stable we can easily argue that a solution for any constant spatially structured input vector $p$ is also stable, since bounded input would lead to bounded output. First we transform the system (3.1) into a stochastic differential equation of the form in the Theorem I:

Let WLOG $p=0$, as discussed above, $f(t, x)=[W F(x)-x]$, and $G$ is a constant diagonal matrix.

Clearly $V(x)$ satisfies the conditions (i) in Theorem I. Now we only need to verify that $L V<0$ for all $x$. Noting here that the $i$-th partial derivative of the $L_{1}$-norm $|x|$ is 1 for $x_{1}>0$ and -1 for $x_{1}<0$, and provided that condition (ii) is satisfied,

$L V=\sum_{i=0}^{N-1}\left|[W F(x)]_{i}\right|-\left|x_{i}\right| \leq \sum_{i=0}^{N-1}\left|\sum_{j=0}^{N-1} \beta w_{j i} x_{j}\right|-\sum_{i=0}^{N-1}\left|x_{i}\right|=\beta\left|x^{T} W\right|-\sum_{i=0}^{N-1}\left|x_{i}\right| \leq$

$\leq \beta|\mathrm{W}||\mathrm{x}|-|\mathrm{x}|<0$

thus completing the proof.

It is interesting to note that the conditions above correspond to the conditions guaranteeing that deterministic network is a contraction mapping and in the case that matrix $W$ is symmetric the condition on the norm can be easily translated into a condition on its maximum eigenvalue (Kelly 1988) 
4. Stability of Linearized Network and Noise Reduction.

In the previous section we exhibit conditions sufficient for statistical stability of stationary state for the sigmoid network. However we view these conditions as somewhat difficult to interpret and therefore we apply local stability analysis to (2.2) with a view to develop more intuitive conditions on the stability of the mean and bounded stationary covariance.

We linearize network (2.2) about the origin and explore the dynamics. Thereby we are studying the local response of the full system to inputs of moderate amplitude. Numerical simulations suggest that even for stimuli with high positive amplitude, that is close to the saturation of the sigmoid, the following analysis provides an upper bound, since the derivative of the sigmoid near its saturation level is below that at the origin. Without loss of generality we set the Jacobian of the sigmoid evaluated at 0 to be identity matrix and study dynamics of linear network described by:

$$
d x=(-x(t)+p+W x(t)) d t+\sigma I d W_{t}
$$

We first show that the mean of (4.1) goes to an asymptotically stable steady state $m_{x}$, provided that the condition on the eigenvalues of the connection matrix $W$ stated below is satisfied.

Noting that the transition matrix for the random process described by $(4.1)$ is

$$
\Phi\left(t-t_{0}\right)=\exp \left((W-I)^{-1}\left(t-t_{0}\right)\right)
$$

we use variation of constants arguments to solve for the mean of the process $m_{x}(t)$ :

$$
m_{x}(t)=e^{(W-1)^{-1}\left(t-t_{0}\right)} x_{0}+(W-I)^{-1} e^{(W-I)^{-1}\left(t-t_{0}\right)} p+(I-W)^{-1} p
$$


Clearly, if all eigenvalues of the connection matrix $W$ have real parts less then 1 , the system tends to a asymptotically attractive steady state in the mean

$$
\text { (4.4) } \mathrm{m}(\infty)=(\mathrm{W}-\mathrm{I})^{-1} \mathrm{p} \text {. }
$$

We now consider the covariance structure. In general the covariance structure for a linear stochastic differential system is given by

$$
K_{x}(t, u)=E\left\{\left[x(t)-m_{x}(t)\right]\left[x(u)-m_{x}(u)\right]^{T}\right\}
$$

Such an equation does not have a closed form solution. However using variation of constants with the transition matrix (4.3) and the fact that the noise term in the equation is modeled with a delta-correlated Gaussian process,

$$
K_{x}(t, u)=\sigma^{2} P D\left(\frac{\lambda_{i}-1}{2}\right) D\left(e^{\left(t+u-t_{0}\right) /\left(\lambda_{i}-1\right)}-e^{(t-u) /\left(\lambda_{i}-1\right)}\right) P^{T}
$$

Where $P$ is a matrix of the orthonormal eigenvectors, $D\left({ }^{*}\right)$ is a diagonal matrix with the diagonal elements as indicated in the parenthesis, and $\lambda_{i}$ is an eigenvalue of matrix W.

If we constrain all the eigenvalues to have real parts below 1 , we see that the exponentials are negative and as $t$ tends to infinity the expression tends to a stationary matrix dependent only on the difference $t-u$. The variance matrix, is found by setting $t=u$ in the above expression:

$$
V_{x}(t)=\sigma^{2} P D\left(\frac{\lambda_{i}-1}{2}\right) D\left(\exp \frac{2 t}{\left(\lambda_{i}-1\right)}-1\right) P^{T}, i=1, \ldots, N
$$

Here the diagonal elements are the variances and the off diagonals are the spatial covariances between the different units. Now if the eigenvalues I are all less than 1 , the second term tends to zero and we are left with 
(4.8) $\mathrm{V}_{\mathrm{x}}(\infty)=\sigma^{2} \mathrm{PD}\left(\frac{1-\lambda_{\mathrm{i}}}{2}\right) \mathrm{P}^{\mathrm{T}}, \mathrm{i}=1, \ldots, \mathrm{N}$

Now we look at a particular example of system (4.1) that is consitent with models of sensory cortical networks exhibiting center on excitation and lateral inhibition. Let identical connection patterns be associated with each unit in the network and let these patterns be symmetric about the center unit, then the system under consideration is homogeneous and symmetric with the connection matrix becoming a circulant. The eigenvectors and the eigenvalues, can be expressed as:

$$
\overrightarrow{\mathrm{x}}^{\mathrm{j}}=\left[\begin{array}{llll}
1 & \mathrm{e}^{2 \pi \mathrm{j} / \mathrm{N}} & \ldots & \mathrm{e}^{2(\mathrm{~N}-1) \pi \mathrm{ij} / \mathrm{N}}
\end{array}\right]^{\mathrm{T}} \quad \lambda_{J}=\sum_{s=0}^{N-1} \mathrm{w}_{s} e^{2 \pi i j s / N}
$$

The mean steady state can now be explicitly calculated according to

(4.10) $\bar{x}_{k}=\sum_{j=0}^{N-1} \frac{\Pi_{j}}{1-\lambda_{j}} e^{2 p_{j} j k / N}$ where $\Pi_{k}=\frac{1}{N} \sum_{k=0}^{N-1} p_{k} e^{-2 \Pi_{i k / N}}$

The variance matrix for such a network can be readily expressed in terms of the elements of the eigenvectors and the corresponding eigenvalues. In fact since the eigen vectors are orthonormal, if we further restrict the eigen values to be positive the stationary variances are less than the variance of the input. That is,

(4.11) $\frac{\sigma^{2}}{2}\left(1-\lambda_{s}\right)<1 \quad \forall s=1, \ldots, N$

and term-by-term the variance matrix obeys the inequality

(4.12) $\left[\mathrm{V}_{\mathrm{x}}(\infty)\right]_{\mathrm{ij}}<\frac{\sigma^{2}}{2}$

Thus we have the following result.

Theorem 3.

Let $p$ be time invariant spatially structured input perturbed at each node of network (4.1) by a stationary delta correlated zero mean noise. 
Let following condition holds for the homogeneous system (4.1)

(i) All eigenvalues of connection matrix $\mathrm{W}$ are less than 1 and positive.

Then the network tends to statistical stationarity. That is:

a. (4.1) has a stationary mean state given by (4.10)

b. The covariance tends to stationarity.

c. The steady state variances (4.11) are less than the variance of the input data.

We now show that the condition above is consistent with near balance of inhibition and excitation in a cortical network. Namely let us consider a connection matrix $W$ where the total sum of on-center excitation and off-center inhibition for each unit is some positive number close to zero and the lateral connection patterns are sparse and weak'. We know from matrix theory that for symmetric circulant or Toeplitz matrices the eigenvalues are given by

$$
\lambda_{j}=\sum_{s=0}^{N-1} W_{s} \cos (2 \pi j s / N)
$$

then the eigen values are bounded above by the sum of the weights. The Gershgorin theorem ${ }^{2}$ states that for a matrix that is diagonal dominant (consistent with diffusely connected network with relatively strong within column excitation and weak sparse pericolumnar inhibition) the eigen values obey

\footnotetext{
1 This is consistent with connectivty observed in anatomical studies of a number of cortical regions (Lund, DeFelipe, Levitt, Mason, Jones, Thompson)

2 Theorem(Gershgorin or Spectral Radius for circulant matrices) (Swartz, 1973)

For a diagonally dominant circulant matrix, the eigen values are contained in a circle of radius given by $\sum_{i \neq j}\left|a_{i j}\right|$, the sum of absolute values of the off-diagonal elements and centered on $\left|a_{i 1}\right|$, the absolute value of the diagonal element.
} 


$$
\left|W_{i i}\right|-\sum_{j \neq i}\left|W_{i j}\right| \leq \max \lambda_{j} \leq\left|W_{i i}\right|+\sum_{j \neq i}\left|W_{i j}\right|
$$

Now lets take a cortical network with excitatory self-connection and inhibitory lateral connectivity, when such a network is near a balance of excitation and inhibition we have $\sum_{s} W_{s} \approx 0$. Let us now further assume that the iinhibiiton is slightly weaker than the excitation (see footnote 1), so that the sum of the weights is positive then conditions for Theorem 3 hold as long as $\sum_{s}\left|W_{s}\right|<1$. Interestingly this implies a network that damps any inputs as opposed to amplifying them and such a property has been recently proposed for the primary somatosensory cortical circuits (Pinto '97).

\section{Discussion.}

In this work we develop analytical conditions that guarantee stable encoding of spatial structure in the presence or random perturbations by a recurrent neural network. A condition on the max-norm of the connection matrix and the derivative of the sigmoid guarantees B.I.B.O. statistical stability of a time-invariant input for a general network. For stimuli that fall into the linear region of the sigmoid we show that a condition on the eigen values of the connection matrix results in stable steady state in the mean and covariance. Such an assumption about the amplitude of the input is not at all unreasonable especially in the view of a recent results by Bell at al (1996) suggesting that for optimal information transfer in the linear region of the sigmoid. For the case of a homogeneous symmetric neural network, of which lateral inhibitory cortical networks are an example, we provide expressions for the mean steady state, the stationary covariance and the eigenvalues. We also present a condition that guarantees that the 
network will reduce the variance of the input upon reaching stationarity. Interestingly, the general condition of section 3 assures the linear results for the symmetric, homogeneous network. Numerical simulations have supported the analytical results. We must point out a caveat; the conditions on the eigenvalues of the connection matrix and the condition in section 3 are only sufficient and we have been able to observe stable encoding by networks that do not satisfy Theorem 2 or 3 . A fruitful future direction would be to study the loss of stability as parametrized by some function of the connection weights and construct the conditions necessary for stable encoding of spatial features by such recurrent neural networks. Furthermore in this paper we do not address stability with respect to noise of temporal structure of a stimulus, but only the stability of spatial structure in a temporally invariant stimulus and we recognise that

much more work needs to be done on the noise reducing properties of recurrent networks viz a vis temporaly structured network.

\section{REFERENCES}

Bell, A.J., Mainen, Z.F., Tsodyks, M., Sejnowski, T.J. (1995) What are the causes of cortical variability?, presented at Neural Dynamics Workshop, Prague.

Buhmann, J., Schulten, K. (1987). Influence of Noise on the Function of a "Physiological" Neural Network. Biological Cybernetics, 56:313-327.

Cohen, M.A, Grossberg, S. (1983) Absolute stability of global pattern formation and parallel memory storage by competitive neural networks. IEEE Transactions on System, Man, and Cybernetics, SMC-13, 815-826. 
Cowan, J. D. (1970). "A statistical mechanics of nervous activity". Lectures on Mathematics in Life Sciences II: Some Mathematical Questions in Biology., Providence, RI, American Mathematical Society.

DeFelipe, J., Hendry, SHC., Hashikawa, T., Molinari, M., Jones, EG. (1990). A microcolumnar structure of monkey cerebral cortex revealed by immunocytochemical studies of double bouquet cells. Neurosci., 37, 655-673.

DeFelipe, J., Farinas, U. (1992). The pyramidal neuron of the cerebral cortex: morphological and chemical chracteristics of the synaptic inputs. Progress in Neurobio., 39, 563-607.

Douglas, R. J., Koch, C., Mahowald, M., Martin, K.A.C., Suarez, H.H. (1995). “Recurrent excitation in neurocortical circuits." Science, 269: 981-985.

Ermentrout, G. B. (1982). Asymptotic Behaviour of stationary homogeneous neuronal nets. Competition and Cooperation in Neuronal Nets. Berlin, Springer-Verlag. Gard, T. C.(1988) Introduction to Stochastic Differential Equations. M. Dekker. Gutkin B. S. And Smith C.E.(1994) On the Stochastic Stability of A Neural Network Model of Somatosensory Information Processing. Neurobiology of Computation, J Bower (ed.), Kluwer.

Jones, E. G. (1981). The Columnar basis of cortical circuitry. In W. D. Willis (Ed.), The Clinical Neurosciences . London: Churchill Livingstone.

Katz, L. C. (1987). Local circuitry of identified projection neurons in cat visual cortex brain slices. J. Nouroscience, 7(4), 1223-1249.

Juliano, S. L., Hand, P.J., Whitsel, B.L. (1981). "Patterns of Increased Metabolic Activity in Somatosensory Cortex of Monkeys Macca fascicularis, Subjected to Controlled Cutaneous Stimulation: a 2-Deoxyglucose Study." Journal of Neurophysiology 46(6): 1260-1284. 
Kelly D.G. (1988) Contractive Nonlinear Neural Networks: Stability and Spatial Frequencies, Institute of Statistics Mimeo Series \#1749, University of North Carolina at Chapel Hill.

Kelly, D. G. (1990). "Stability in contractive nonlinear neural networks." IEEE Trans. Biomed. Eng. 37(3): 231.

Kishimoto, K., Amari, S. (1979) Existence and stability of local excitations in homogeneous neural fields. J. Math. Biol. 7: 303-318

Levitt, J. B., Lewis, D.A., Yoshioka, T., Lund, J.S. (1993). Topography of pyramidal neuron intrinsic connections in macaque monkey prefrontal cortex (Area 9 and 46). J.Comp. Neurol., 338, 360-376.

Lund, J. S., Lewis, D.A. (1993). Local circuit neurons of developing and mature macaque prefrotnal cortex: Golgi and immunocytochemical characteristics. J. Comp. Neur., 328, 282-312.

Mason, A., Nicoll, A., Stafford, K. (1991). synaptic transmission between individual pyramidal neurons of the rat visual cortex in vitro. J. Neurosci., 11(1), 72-84.

Ohura T., Cowan J.D. (1995) Stochastic Single Neurons, Nerual Computation 7(3):518-529

Pinto, D. (1997) Doctoral Dissertation, Dept of Mathematics, University of Pittsburgh, Pittsburgh Pa.

Shama, S. (1989). Spatial and Temporal Processing in Central Auditory Networks. In C. Koch Segev, Igdan (Eds.). Methods in Neuronal Modelling: From Synapses to Networks. Cambridge, Mass: MIT Press. 
Smith, C. E., Kelly, D.G. (1985). "Simulations of a Spatiotemporal Model for Cortical 2DG Pattern". Abstract \#264.14, American Society for Neuroscience 15th Annual Meeting, Dallas, Texas.

Swartz, H.R. (1973) Numerical Analysis of Symmetric Matrices, Prentice-Hall, Edgewood Cliffs, NJ.

Thomson, A. M., Deuchars, J. (1994). Temporal and spatial properties of local circuits in neocortex. TINNS, 17(3), 119-126.

Whitsel, B., Kelly, D. (1988). Knowledge Acquisition ("Learning") by the Somatosensory Cortex. Brain Structure. Learning, and Memory. Westview Press. 93-127. 


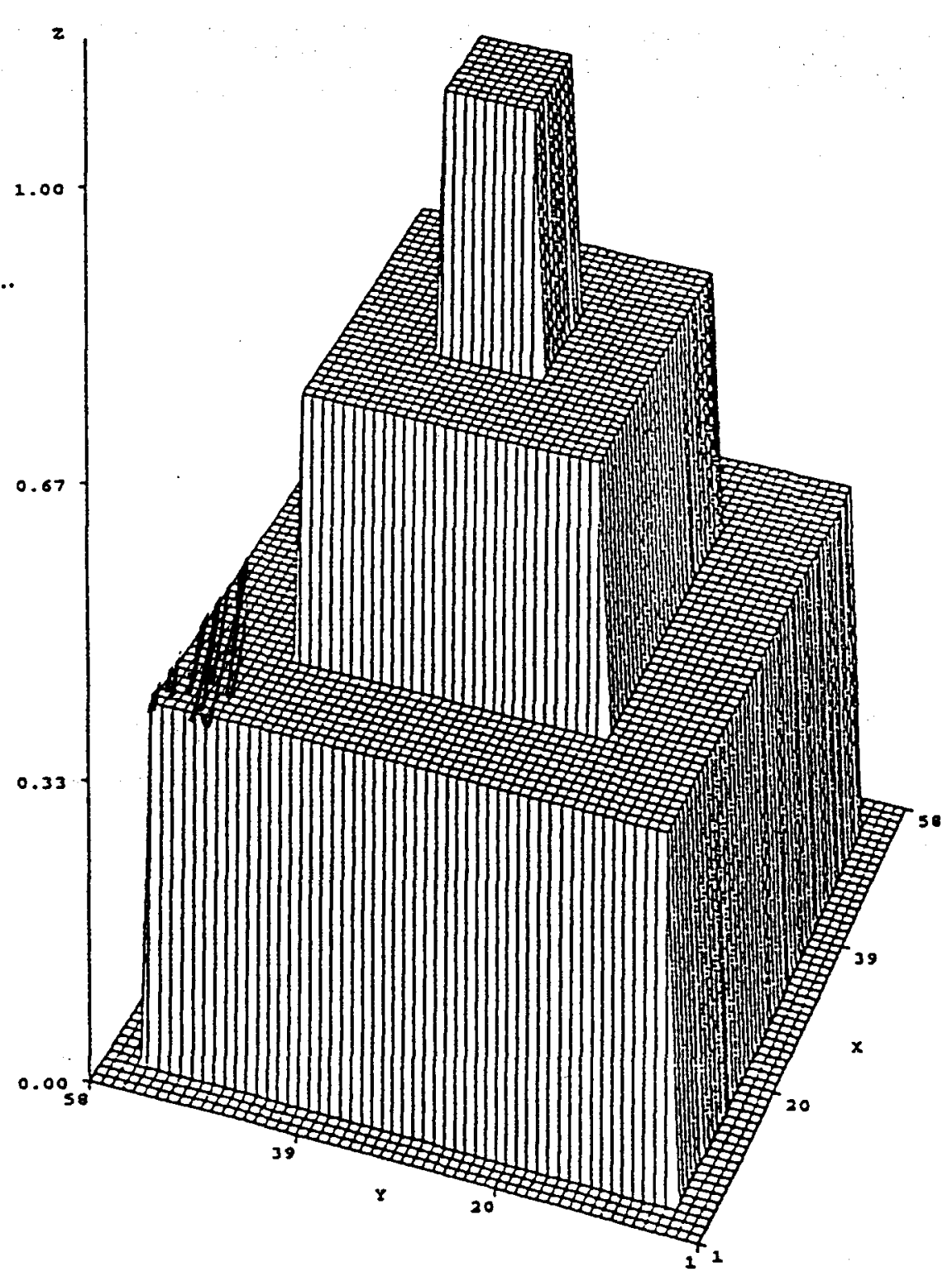

Fig. 1 STIMULUS INPUT 


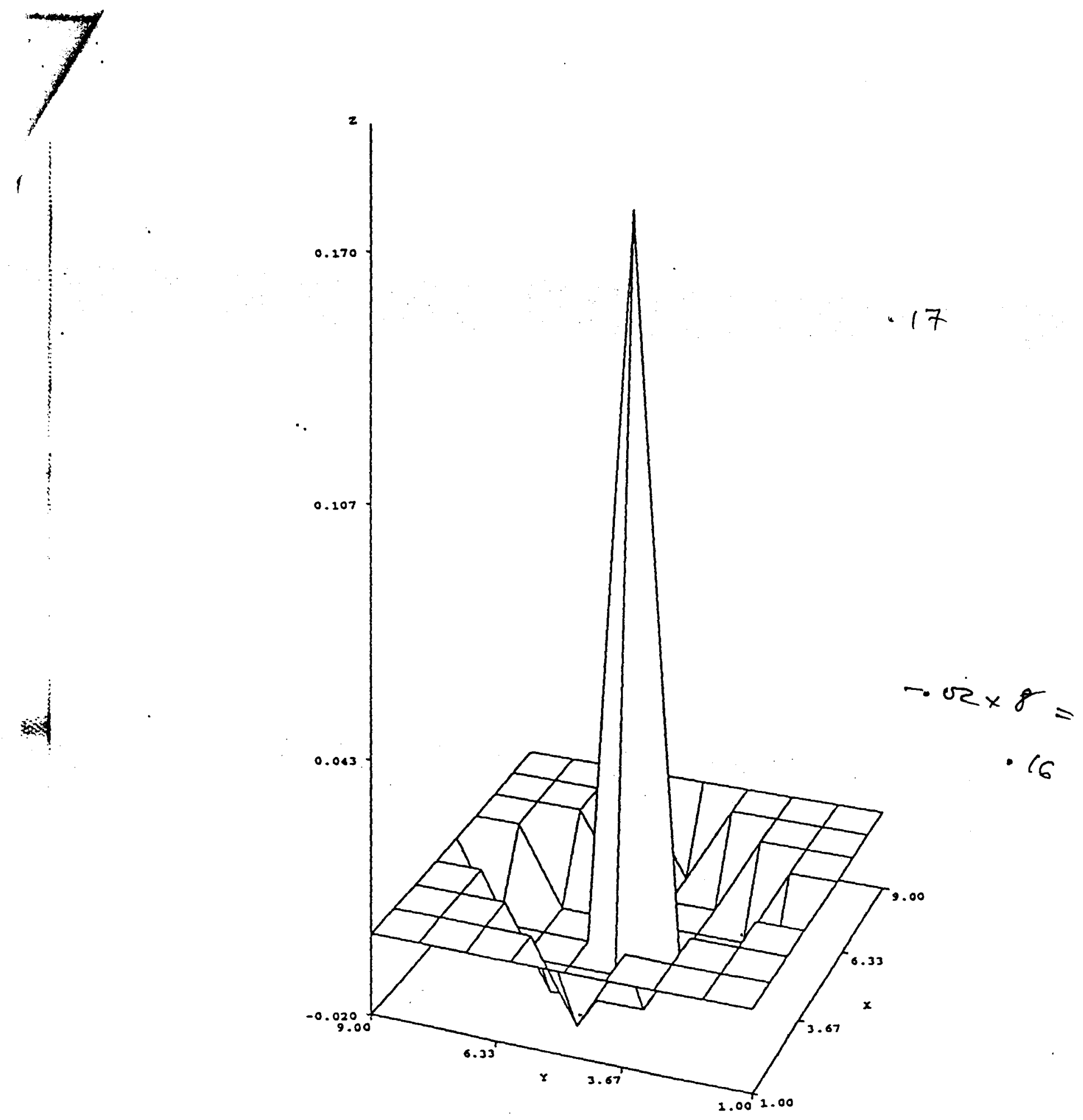

Fig. Ba. CONNECTION PATTERN \#1 


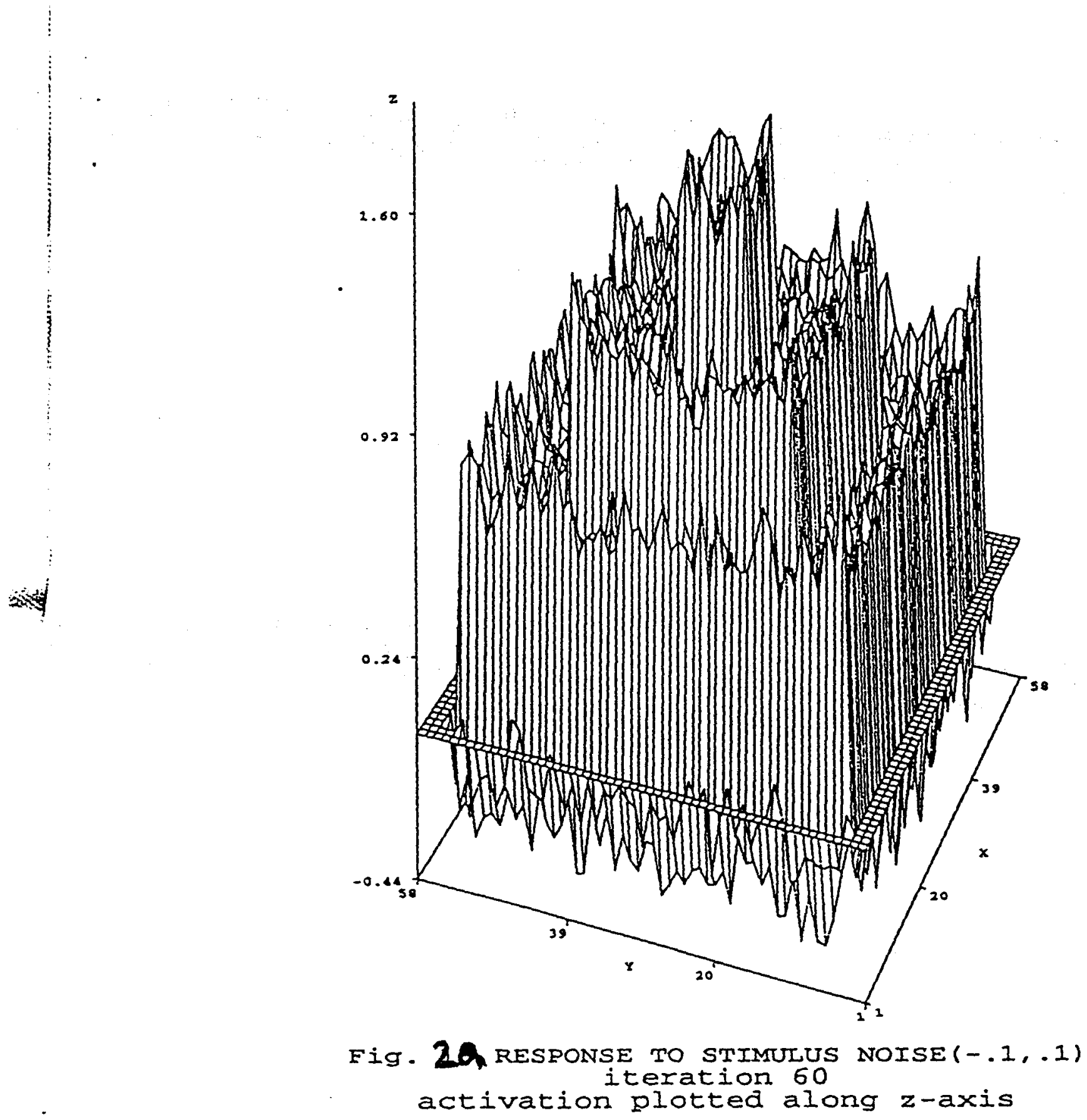




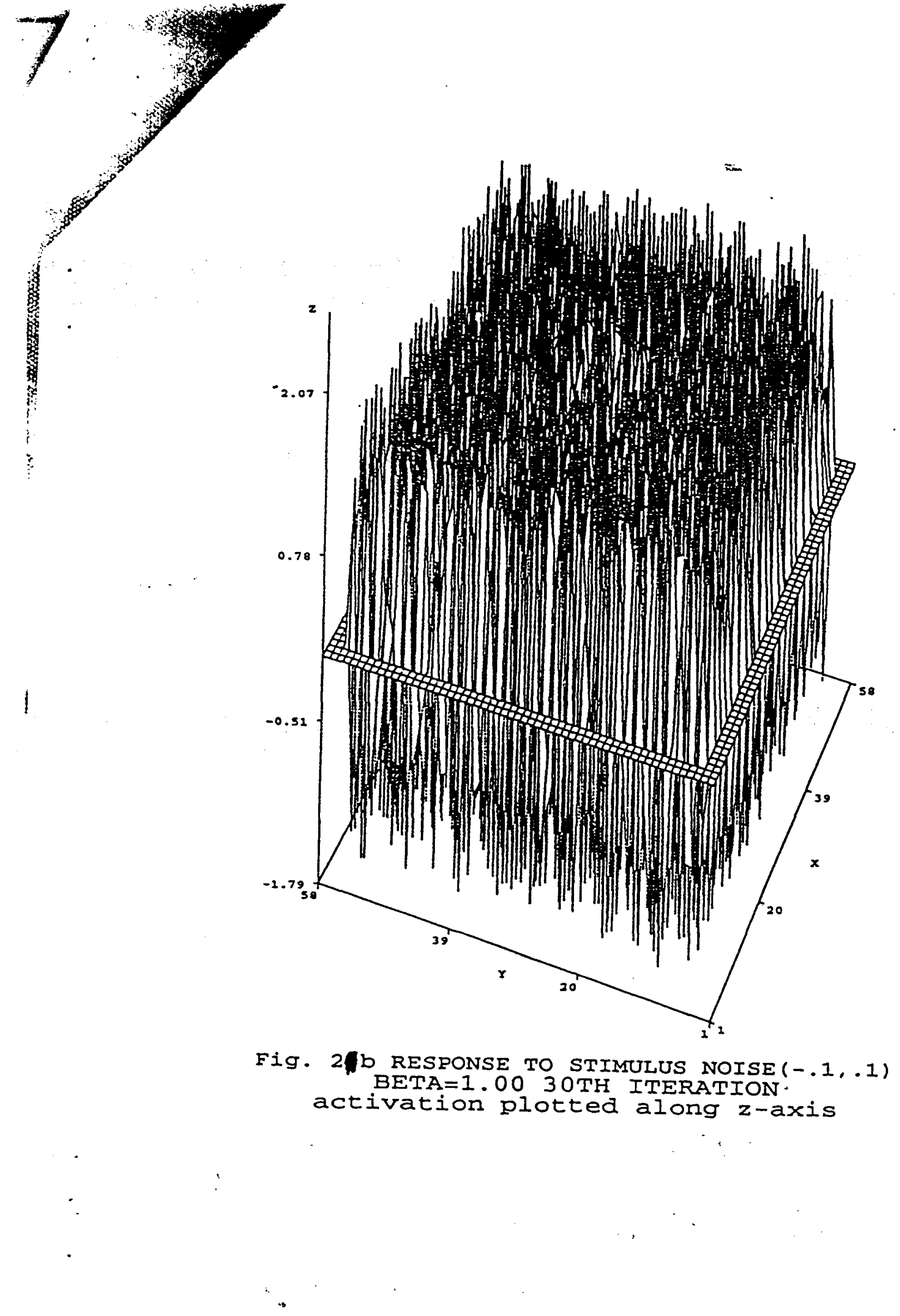

